\title{
Development of a Polystyrene Reference Material for Raman Spectrometer (NMIJ RM 8158-a)
}

Nobuyasu ITOH ${ }^{* \dagger}$ and Nobuyasu HANARI*

* National Metrology Institute of Japan (NMIJ), National Institute of Advanced Industrial Science and Technology (AIST), Central 3, 1-1-1 Umezono, Tsukuba, Ibaraki 305-8563, Japan

$\dagger$ To whom correspondence should be addressed.

E-mail: nobuyasu-itoh@aist.go.jp 


\begin{abstract}
The Raman shift is one of the most important parameters in Raman spectroscopy, and is calculated from the difference in the wavenumbers for excitation and for Raman scattering. Because the observed shifts are strongly dependent on the spectrometer and the measurement conditions, physically and chemically stable reference materials for Raman-shift validation are required. We reliably estimated the Raman shift and evaluated its uncertainty for peaks of a 4-mm-thick, 25-mmdiameter polystyrene disc by using a HeNe laser and Raman-scattered light calibrated with $\mathrm{Ne} / \mathrm{Ar}$ emission lines. We considered uncertainties originating from the fitting, repeatability, and reproducibility of both the $\mathrm{Ne} / \mathrm{Ar}$ emission lines and the Rayleigh/Raman-scattered lights, as well as the wavenumber of the unstabilised HeNe laser, and pixel and spectral resolutions. We also considered uncertainty originating from inhomogeneity among and within discs, as well as their long-term stability. The obtained Raman shifts of 11 peaks (reference values) were comparable to those described in ASTM E1840 with expanded uncertainty at $1.1 \mathrm{~cm}^{-1}$ or $1.2 \mathrm{~cm}^{-1}(k=2$, coverage factor).
\end{abstract}

Keywords: Reference material, polystyrene, Raman shift, HeNe laser, emission lines, uncertainty 


\section{Introduction}

The Raman shift originates from the difference between the energy of excitation and that of Raman scattering. ${ }^{1-4}$ Although Raman shifts are theoretically independent of the Raman spectrometer that is used, in practice they are markedly dependent upon it. Consequently, users must calibrate individual Raman spectrometers by using references or protocols provided by manufacturers, because no standardized protocols are available for calibration.

Wavelengths/wavenumbers of elemental emission lines, such those of Ne and Ar, are suitable for accurate calibration of Raman spectrometers; however, they are not suitable for daily calibration of some Raman systems. For most applications of Raman systems, Raman-shift values of wellcharacterized materials provided in ASTM E1840 are more convenient and more easily handled. ${ }^{1-3,5}$ However, Raman shifts of the materials provided in ASTM E1840 are not associated with the corresponding uncertainty information, which is essential for obtaining reliable values. ${ }^{5,6}$

Polystyrene, which is described in ASTM E1840 and in which the Raman shifts of its 11 peaks are provided, ${ }^{5}$ has been widely used as a reference material because it is not only physically and chemically stable, but is also free from any regulations related to chemical reagents. Polystyrene is therefore a suitable material to be developed as a reference material for the validation of Raman shifts in accord with ISO Guide 35:2017.7

We have reliably estimated the Raman shift of the 11 peaks of polystyrene by using wavenumbers of a HeNe laser and Raman-scattered lights calibrated with $\mathrm{Ne} / \mathrm{Ar}$ emission lines to develop a reference material (NMIJ RM 8158-a, Polystyrene for Raman spectrometer). We have also validated the reference values with other systems and by means of interlaboratory comparisons.

\section{Experimental}




\section{Sample Preparation}

The candidate material, a polystyrene disc with a diameter of $25 \mathrm{~mm}$ and a thickness of 4 mm, was prepared by injection-moulding of polystyrene pellets (HF77; PS Japan, Tokyo, Japan) followed by surface processing to give one side a mirror finish while the other side had a rough surface. Each disc was packed in an aluminium-laminated plastic bag under nitrogen gas.

\section{Wavenumbers and Their Uncertainties Referred in This Study}

The wavelength in vacuum $(\lambda=632.9908 \mathrm{~nm})$, frequency $(f=473.6127 \mathrm{THz})$, and relative standard uncertainty $\left(1.5 \times 10^{-6}\right)$ of an unstabilised HeNe laser, as recommended by the Comité International des Poids et Mesures (CIPM), are reliable and SI traceable. ${ }^{8,9}$ In this study, we used the wavenumbers of the HeNe laser and the $\mathrm{Ne} / \mathrm{Ar}$ emission lines in vacuum, and their corresponding uncertainties. The wavenumber of the HeNe laser in vacuum $\left(15798.018 \mathrm{~cm}^{-1}\right)$ was calculated from the reciprocal of its wavelength in vacuum. Wavenumbers of the Ne emission lines in vacuum were taken from the report by Saloman and Sansonetti, ${ }^{10}$ and their uncertainties were calculated from the relative uncertainty of wavelengths in air. Wavenumbers of the Ar emission lines in vacuum were taken from the report by Whaling et al. ${ }^{11}$ after correction according Sansonetti, ${ }^{12}$ whereas their uncertainties were used without any correction.

\section{Characterization of Raman Shifts}

The Raman spectrometer (LabRAM HR Evolution; Horiba, Kyoto, Japan) that we used was equipped with a HeNe laser (laser power: 4.0 mW), a 10× objective lens (numerical aperture: 0.25), and a $600 \mathrm{gr} / \mathrm{mm}$ grating, and its pinhole size was set at $100 \mu \mathrm{m}$ diameter. Peak positions were calibrated by using light from a Ne or Ar reference lamp (UVP Pen-Ray Rare Gas Lamps; Analytik Jena $\mathrm{GmbH}$, Jena, Germany) inserted through a beam splitter set in front of the objective lens.

Figure 1 shows the Raman spectrum of a polystyrene disc from 500 to $3200 \mathrm{~cm}^{-1}$ under the 
same spectral pixel resolutions with Raman shift values provided in $A S T M E 1840,{ }^{5}$ together with an assignment of the identities of the peaks used in this study. Although all peaks should be originated from polystyrene itself, we only evaluated 11 peaks which is provided in ASTM E1840. ${ }^{5}$ To estimate the wavenumbers of the peaks, including the Rayleigh scattering of the HeNe laser, data were separately obtained in 12 spectral windows [see the Electric Supplementary Information (ESI), Figs. S1-S3]. After obtaining data for the Ne/Ar emission lines, data for the Rayleigh- and Raman-scattered lights were obtained without moving the grating. The grating was then moved and sets of data for the next spectral window were obtained. Detailed information on the measurements and the spectral windows are summarized in the ESI (Tables S1 and S2).

A mixed Gaussian-Lorentzian function was used to estimate the wavenumbers of all the peak-tops for the Ne/Ar emission lines and for the Rayleigh- and Raman-scattered lights. Peaks F, J, and $\mathrm{K}$ were fitted as one bilaterally symmetric peak in each case, even though those peaks are bilaterally asymmetric (Fig. 1).

Changes in peak-top values for Peaks $\mathrm{F}, \mathrm{J}$ and $\mathrm{K}$, originating from different spectral resolutions, were evaluated by changing both the pinhole size $(100,200,400,600,800$, and $1000 \mu \mathrm{m}-$ $\varphi)$ and the grating density $(300,600,1200$, and $1800 \mathrm{gr} / \mathrm{mm})$ for a $532 \mathrm{~nm}$ laser. Uncertainty originating from changes in the spectral resolution were evaluated from the variance of peak-top values in 21 different spectral resolutions (spectral resolution range examined: $2.0-22 \mathrm{~cm}^{-1}$; spectral resolution at characterization: $3.7 \mathrm{~cm}^{-1}$ for the calcite peak at $1085 \mathrm{~cm}^{-1}$ ).

\section{Overview of Characterization of Raman Shift Values and Their Uncertainty}

For a reliable estimation of the wavenumbers of the HeNe laser and the Raman-scattered lights, we applied the following procedure and we considered several factors for the evaluation of the expanded uncertainty. Here, we also used the estimated wavenumber of the HeNe laser with $\mathrm{Ne}$ emission lines as Raman-scattered lights, because possible systematic changes in measurements can 
be cancelled out by using the wavenumbers obtained under the same calibration conditions.

1. The wavenumbers of $\mathrm{Ne}$ and Ar emission lines, together with their uncertainty, reported in the literature $^{10-12}$ were used to calibrate wavenumbers and to evaluate their uncertainty.

2. A third-order polynomial calibration curve $\left(y=a+b x+c x^{2}+d x^{3}\right)$ was obtained from more than five $\mathrm{Ne} / \mathrm{Ar}$ emission line wavenumbers reported in the literature (in vacuum) and their observed wavenumbers as peak-tops (in air) for each spectral window.

3. Wavenumbers of both the Raman-scattered lights and the HeNe laser in vacuum were estimated by using the third-order polynomial calibration curve $\left(y=a+b x+c x^{2}+d x^{3}\right)$ obtained for each spectral window (without moving the grating).

4. Wavenumbers of the HeNe laser and of each of the Raman-scattered lights obtained from more than three different spectral windows (regions of grating) for each peak were combined. Raman shift values were then estimated by subtracting the combined wavenumber of the Raman-scattered lights from that of the HeNe laser.

We also considered the uncertainty of the peak fitting, the repeatability and reproducibility of the peak-top estimation separately evaluated by 5 iteration for each peak at each window, and differences in regions of the spectral window and in the spectral and pixel resolutions, as well the as uncertainty for the wavenumber of the HeNe laser.

\section{Homogeneity and Stability}

The homogeneity among the polystyrene discs was assessed by estimating the Raman shifts at two points for ten discs, hierarchical-randomly selected from among 1900 discs according to ISO Guide $35: 2017 .^{7}$ The homogeneity within a disc was also assessed by measuring at ten randomly selected points on the disc and calculating the standard deviations of the measurements for these points. The long-term stability of polystyrene discs stored at $15^{\circ} \mathrm{C}$ to $25^{\circ} \mathrm{C}$ in darkness was assessed by estimating the Raman shifts of all peaks for two points on each disc at 1,2, 4, 6, 8, and 9.5 months. 
More details are given in the ESI.

Validation of the Raman-Shift Characterization and Reference Values

We used an acetaminophen [GBW 3654; paracetamol, $N$-(4-hydroxyphenyl)acetamide] certified reference material (CRM) obtained from the National Metrology Institute of China (NIM), ${ }^{13}$ and we examined 17 peaks between 500 and $3100 \mathrm{~cm}^{-1}$ to validate the Raman-shift values obtained by our characterization method (ESI; Table S3).

Raman shifts of polystyrene peaks were also estimated in the following four ways, and their values were used for additional validation of the reference values:

(a) by using the theoretical wavenumber of the HeNe laser to calculate the Raman shifts,

(b) by using a diode-pumped solid-state (DPSS) laser at $532 \mathrm{~nm}$,

(c) by using an $\mathrm{Ar}^{+}$laser at $488 \mathrm{~nm}$, and

(d) by an interlaboratory comparison for 42-50 different sets of models and analytical conditions.

Measurement conditions and other detailed information are provided in the ESI.

\section{Results and Discussion}

\section{Observed wavenumbers of the HeNe laser and the Raman-Scattered Lights}

To obtain reliable estimates of the wavenumbers of the HeNe laser and the Raman-scattered lights, we measured 10 discs for characterization and obtained wavenumber in air for each peak at each window and evaluated each reproducibility.

Table 1 shows the observed wavenumbers of the HeNe laser and the Raman-scattered lights for each spectral window. Here, the uncertainty $(u)$ was estimated from the standard deviation divided by $\sqrt{10}$ because ten samples were used in the characterization. The uncertainty $(u)$ of the wavenumber for each window was ranged from $0.0362 \mathrm{~cm}^{-1}$ to $0.2215 \mathrm{~cm}^{-1}$, and differences in the observed 
wavenumbers for each window ranged from $0.0128 \mathrm{~cm}^{-1}$ to $0.1457 \mathrm{~cm}^{-1}$. Our results suggested that the reproducibility of the observed wavenumbers of the HeNe laser and the Raman-scattered light for each window was good and that differences in the wavenumbers measured in different region of the grating (spectral window) were small.

Evaluation of the Third-Polynomial Calibration Curve and its Uncertainty by Using Wavenumbers of the Ne/Ar Emission Lines

To estimate the wavenumbers of the HeNe laser and the Raman-scattered lights in vacuum, third-polynomial calibration curves were obtained from the wavenumbers of the Ne/Ar emission lines provided in the literature (in vacuum) and those observed with our system (in air). ${ }^{10-12}$ The thirdpolynomial calibration curve at each window and its uncertainty was estimated from Eq. 1 below:

$$
\begin{aligned}
& Y_{\text {Ref.,i }}^{N e / A r}=a_{i}+b_{i} \times\left(X_{\text {Measure }, i}^{\mathrm{Ne} / A r} \times F_{\text {Fitting }, i}^{\mathrm{Ne} / A r} \times F_{\text {Repeat }, i}^{\mathrm{Ne} / A r} \times F_{\text {Repro.,i }}^{\mathrm{Ne} / A r}\right) \\
& +c_{i} \times\left(X_{\text {Measu }}^{\mathrm{Ne} / \mathrm{Ar}} \times{ }_{i} \times F_{\text {Fitting }, i}^{\mathrm{Ne} / \mathrm{Ar}} \times F_{\text {Repeat }, i}^{\mathrm{Ne} / \mathrm{Ar}} \times F_{\text {Repro. }, i}^{\mathrm{Ne} / A r}\right)^{2} \\
& +d_{i} \times\left(X_{\text {Measure }, i}^{\text {Ne/Ar }} \times F_{\text {Fitting }, i}^{\text {Ne/Ar }} \times F_{\text {Repeat }, i}^{\text {Ne/Ar }} \times F_{\text {Repro }, i}^{\text {Ne/Ar }}\right)^{3}
\end{aligned}
$$

where $Y_{R e f, i}^{N e / A r}$ is the wavenumber of the Ne/Ar emission line in vacuum reported in the literature, ${ }^{10-}$ ${ }^{12} X_{\text {Measure }, i}^{\mathrm{Ne} / \mathrm{Ar}}$ is the observed wavenumber of the Ne/Ar emission line in air, $F_{F i t t i n g, i}^{\mathrm{Ne} i \mathrm{Ar}}$ is the variance of fitting for the Ne/Ar emission line calculated by using the mixed Gaussian-Lorentzian function (typical value =1), $F_{\text {Repeat }, i}^{N e / A r}$ is variance of repeatability without movement of grating for the $\mathrm{Ne} / \mathrm{Ar}$ emission line (typical value $=1$ ), and $F_{R e p r o ., i}^{N e / A r}$ is variance of the repeatability with movement of the grating for the Ne/Ar emission line (typical value =1). $F_{F i t t i n g, i}^{N e / A r}, F_{R e p e a t, i}^{N e / A r}$, and $F_{\text {Repro., } i}^{N e / A r}$ were evaluated separately for each peak in each window and considered for uncertainty of calibration curve. The subscript $i$ indicates the window number. The uncertainty of $Y_{R e f ., i}^{N e / A r}\left[u\left(Y_{R e f ., i}^{N e / A r}\right)\right]$ was obtained from the literature, ${ }^{10-12}$ and the uncertainty of $X_{\text {Measure, } i}^{\text {Ne/Ar }}\left[u\left(X_{\text {Measure, } i}^{\mathrm{Ne} / \text { Ar }}\right)\right]$ was obtained from reproducibility $(n=10)$ of observed wavenumbers in air for characterization. Uncertainty of coefficients $a_{i}, b_{i}, c_{i}$ and $d_{i}$ were calculated by means of a weighted Deming regression using the 
typical values with uncertainties. Here, coefficients $a_{i}, b_{i}, c_{i}$ and $d_{i}$ obtained using a weighted Deming regression were the same with those obtained with a least squares method because considered uncertainty were quite smaller than the values. Thus, we used the least regression method for estimation of calibration curve in each window.

Tables $2 \mathrm{a}$ and $2 \mathrm{~b}$ summarize the values of $Y_{R e f ., i}^{N e / A r}$ and $u\left(Y_{R e f ., i}^{N e / A r}\right)$ obtained from the literature, ${ }^{10-12}$ together with $X_{\text {Measure }, i}^{N e / A r}, F_{\text {Fitting }, i}^{N e / A r}, F_{\text {Repeat }, i}^{N e / A r}, F_{R e p r o, i}^{N e / A r}$, and their combined uncertainty $\left[u c\left(X_{\text {Measure, },}^{N e / A r}\right)\right]$ at Windows 2 and 5 as examples. In most cases, $u\left(X_{\text {Measure }, i}^{N e / A r}\right)$ and $F_{R e p r o ., i}^{N e / A r}$ were the dominant factors, and values of $u\left(Y_{\text {Measure.,i }}^{N e / A r}\right)$ were less than $0.14 \mathrm{~cm}^{-1}$ in all windows.

Estimation of Wavenumbers and Their Uncertainty for the HeNe Laser and the Raman-Scattered Lights by Using the Third-Polynomial Calibration Curve

The wavenumbers of HeNe laser and Raman-scattered lights were calculated from Eq. 2 below:

$$
\begin{aligned}
Y_{\text {Calculation }, i}^{\text {HeNe/Raman }}= & a_{i}+b_{i} \times\left(X_{\text {Measure }, i}^{\text {HeNe/Raman }} \times F_{\text {Fitting }, i}^{\text {HeNe } / \text { Raman }} \times F_{\text {Repeat }, i}^{\text {HeNe } / \text { Raman }} \times F_{\text {Repro }, i}^{\text {HeNe } / \text { Raman }}\right) \\
& +c_{i} \times\left(X_{\text {Measure }, i}^{\text {HeNe } / \text { Raman }} \times F_{\text {Fitting }, i}^{\text {HeNe } / \text { Raman }} \times F_{\text {Repeat }, i}^{\text {HeNe }, \text { Raman }} \times F_{\text {Repro }, i}^{\text {HeNe }, \text { Raman }}\right)^{2} \\
& +d_{i} \times\left(X_{\text {Measure }, i}^{\text {HeNe } / \text { Raman }} \times F_{\text {Fitting }, i}^{\text {HeNe/Raman }} \times F_{\text {Repeat }, i}^{\text {HeNe } / \text { Raman }} \times F_{\text {Repro }, i}^{\text {HeNaman }}\right)^{3}
\end{aligned}
$$

where $Y_{\text {Calculation }, i}^{\text {HeNe/Raman }}$ is the wavenumber of the HeNe laser or the Raman-scattered light in vacuum, $X_{\text {Measure, } i}^{\text {HeNe/Raman }}$ is the observed wavenumber of the HeNe laser or the Raman-scattered light in air, $F_{\text {Fitting,i }}^{\text {HeNe/Raman }}$ is the variance of fitting for the HeNe laser or the Raman-scattered light calculated by using the mixed Gaussian-Lorentzian function (typical value =1), $F_{\text {Repeat }, i}^{\text {HeNe/Raman }}$ is the variance of repeatability without movement of the grating for the HeNe laser or the Raman-scattered light (typical value = 1), and $F_{\text {Repro.,i }}^{\text {HeNe/Raman }}$ is the variance of repeatability with movement of the grating for the HeNe laser or the Raman-scattered light (typical value =1). $F_{\text {Fitting }, i}^{\text {HeNe/Ran }}, F_{\text {Repeat }, i}^{\text {HeNe/Raman }}$, and $F_{\text {Repro,i }}^{\text {HeNe/Raman }}$ were evaluated separately for each peak in each window and considered for uncertainty 
of the observed wavenumber. The subscript $i$ is the window number. Coefficients $a_{i}, b_{i}, c_{i}$ and $d_{i}$ were obtained from Eq. 1. The uncertainty of $X_{\text {Measure, } i}^{\text {HeNe/Raman }}\left[u\left(X_{\text {Measure,i }}^{\text {HeNe/Raman }}\right)\right]$ was obtained from the reproducibility $(n=10)$ of calibrated wavenumbers in vacuum using a third-order polynomial calibration curve at each window for characterization (the same values in Table 1). $Y_{\text {Calculation, } i}^{\text {HeNe/Ran }}$ and its uncertainty $\left[u\left(Y_{\text {Calculation }, i}^{\text {HeNe/Raman }}\right)\right]$ were calculated by a spreadsheet method.

Tables $3 \mathrm{a}$ and $3 \mathrm{~b}$ summarize the budget sheet for $u\left(Y_{\text {Calculation, } i}^{\text {HeNe/Raman }}\right)$ for the HeNe laser (Windows 1, 2, and 3) and for Peak C (Windows 5, 6, and 7), respectively. Same as the Ne/Ar emission lines (Tables $2 \mathrm{a}$ and $2 \mathrm{~b}), u\left(X_{\text {Measure, }}^{\text {HeNe/Raman }}\right)$ and $F_{\text {Repro.,i }}^{\text {HeNelRan }}$ are larger than $F_{\text {Fitting,i }}^{\text {HeNe/Raman }}$ and $F_{\text {Repeat }, i}^{\text {HeNe/Raman }}$, and the wavenumbers of the HeNe laser and the Raman-scattered lights obtained with uncertainty were also comparable among the windows. The same trends were observed for all the other peaks (data not shown).

\section{Evaluation of Uncertainty for the Combined Wavenumber of Each Peak}

Wavenumbers of peaks obtained at three or four windows were combined with the weighted means by using $1 / u_{\mathrm{i}}$, where $u_{\mathrm{i}}$ is the uncertainty of each window, as the weighting factor. The uncertainty in the between-window variance $\left(u_{b w}\right)$ was calculated by performing an analysis of variance (ANOVA) on the results listed in Table 1, and this was taken into consideration if significant. The combined standard uncertainties $\left(u_{c}^{\text {Window }}\right)$ obtained from different windows $\left(u_{\text {Window }}\right)$ and $u_{b w}$ are summarized in Table 4. Eight peaks among the 12 peaks including the HeNe laser were significant and their $u_{b w}$ was taken into consideration in their combined standard uncertainty.

For the combined wavenumbers of peaks, we also considered uncertainty originating from the wavenumber of the HeNe laser itself $\left(u_{\mathrm{HeNe}}\right)$ and from the pixel and spectral resolutions $\left(u_{\text {Pixel }}\right.$ and $u_{\text {Resolution }}$ ). The uncertainty given by the CIPM (relative uncertainty: $1.5 \times 10^{-6}$ ) was used for the uncertainty of the wavenumber of the HeNe laser itself. ${ }^{8,9}$

Because peak-top wavenumbers were estimated by using the mixed Gaussian-Lorentzian 
function, their reliability was also dependent on the pixel resolution. We therefore also considered the pixel resolution for all peaks, although this might be an overestimation for the standard uncertainty. Uncertainty originating from the pixel resolution $\left(u_{\text {Pixel }}\right)$ was evaluated from the rectangular distributions of the half-width of two points (e.g., pixel resolution at the HeNe laser: $1.295 \mathrm{~cm}^{-1} \div 2$ $\div \sqrt{3}=0.3738 \mathrm{~cm}^{-1}$ ), because the pixel resolution can be taken as the point-to-point resolution. The resulting values of $u_{\text {Pixel }}$ ranged from 0.2401 to $0.3738 \mathrm{~cm}^{-1}$ (Table 4).

We also considered the spectral resolutions of asymmetric peaks that probably consisted of several sub-peaks, such as Peaks F, J, and K (Fig. 1), not only because these are probably dependent on such measurement conditions as the wavelength of the laser, the grating density, the focal length, the objective lens, and the pinhole/slit sizes, but also because we regarded these peaks as symmetrical peaks for the mixed Gaussian-Lorentzian function. To evaluate the uncertainty originating from changes in the spectral resolution $\left(u_{\text {Resolution }}\right)$, we examined the variance of spectral resolutions by using 21 different analytical conditions. The resulting uncertainties were $0.1021 \mathrm{~cm}^{-1}, 0.0982 \mathrm{~cm}^{-1}$, and $0.0671 \mathrm{~cm}^{-1}$ for Peaks F, J, and $\mathrm{K}$, respectively, which are much smaller than that of $u_{\text {Pixel }}$ (Table 4).

\section{Estimation of the Raman Shift for Each Peak and Its Expanded Uncertainty}

The Raman shift for each peak can be calculated from the differences in the wavenumbers of HeNe laser and Raman-scattered light, and uncertainty of Raman shift $u_{\text {Char }}$ can also be calculated from wavenumbers' uncertainties of the HeNe laser and Raman-scattered lights in Table 4.

ISO Guide 35:2017 specifies that uncertainties in CRMs should be evaluated from the standard uncertainty due to characterization $u_{C h a r}$, homogeneity of the material $u_{b d}$, standard uncertainty due to long-term instability $u_{l t s}$, and short-term instability (instability during transportation) $u_{s t s} \cdot{ }^{7}$ In the present case, we also considered inhomogeneity within a disc $u_{w d}$, because the point size for characterization $(\sim 10 \mu \mathrm{m})$ was considerably smaller than the disc size $(25$ 
$\mathrm{mm}$ diameter), and there is a possibility of inhomogeneity over a disc. Uncertainty related to longterm stability $\left(u_{l t s}\right)$ was evaluated by ANOVA as $s_{l t s}$, because linearity regression was insignificant. The combined standard uncertainty was calculated by using Eq. 3:

$$
u_{c}=\sqrt{u_{C h a r}+u_{b d}+u_{w d}+u_{l t s}}
$$

Figure 2 summarizes the uncertainty components and their contribution for the combined standard uncertainty as well as Raman shift of each peak. Among the uncertainties considered, $u_{\text {Char }}$ was the dominant factor for all peaks and it was mainly originated from $u_{\text {Pixel }}$. Thus, $u_{\text {Char }}$ and resultant $u_{c}$ decreased gradually with increase of Raman shift (decrease of Raman scattering light's wavenumber).

Table 5 lists the reference values and their expanded uncertainties. The expanded uncertainty $(U)$ in the certified values is equal to $k u_{\mathrm{c}}$, where $u_{\mathrm{c}}$ is the combined standard uncertainty with a coverage factor $k=2$, approximating a 95\% confidence interval. The obtained values were comparable to those described in $A S T M E 1840^{5}$ and their uncertainties were commonly $1.1 \mathrm{~cm}^{-1}$ or $1.2 \mathrm{~cm}^{-1}$.

\section{Validation of the Reference Values by Using Other Approaches}

To validate the reference values and their uncertainty, we applied an estimation of the Raman shift with the reliable wavenumber of the HeNe laser calculated from its wavelength as recommended by CIPM $\left(15798.018 \mathrm{~cm}^{-1}\right)$, estimations with both a $532 \mathrm{~nm}$ DPSS laser and an $\mathrm{Ar}^{+}$ laser, and interlaboratory comparison without any indication of the measurement procedure. The reference values and their uncertainties were comparable to those obtained by other methods, except for the mean value with interlaboratory comparison for Peak I (ESI; Table S4). We therefore believe that the reference values are reasonable and reliable.

We also examined the consistency of the reference values with those described in ASTM $E 1840^{5}$ by using the following equation: 


$$
E_{n}=\frac{\left|R S_{R e f}-R S_{A S T M}\right|}{\sqrt{U_{R e f}^{2}+U_{A S T M}^{2}}}
$$

where $R S_{R e f}$ is the reference value of the reference material, $R S_{A S T M}$ is the Raman shift value given in $A S T M E 1840, U_{R e f}$ is the expanded uncertainty (coverage factor $k=2$ ) of $R S_{R e f}$, and $U_{A S T M}$ is the expanded uncertainty (coverage factor $k=2$ ) of $R S_{A S T M}$, which is the twice the standard deviation described in $A S T M E 1840 .^{5}$ This evaluation showed that the difference is insignificant when $E_{n} \leq 1$. The $E_{n}$ values obtained ranged from 0.10 to 0.67 , and only the value of 1.3 for Peak F was greater than 1 (ESI; Table S5); this was attributed to the method used to decide the peak top (obtained by using the mixed Gaussian-Lorentzian function for this asymmetric peak). Because the reference values for 10 of the 11 peaks were consistent with the ASTM values, it can be considered that the reference values and the ASTM values are consistent with one another.

\section{Conclusions}

A polystyrene disc reference material for Raman spectrometer has been developed by using combination of HeNe laser and Ne/Ar emission lines. Raman shifts of 11 peaks with uncertainties of $1.1 \mathrm{~cm}^{-1}$ or $1.2 \mathrm{~cm}^{-1}$ were reliably obtained from the differences in wavenumbers of the HeNe laser and the Raman-scattered lights calibrated with $\mathrm{Ne} / \mathrm{Ar}$ emission lines, and validated by other four approaches. This polystyrene disc is now available from the National Metrology Institute of Japan (NMIJ) as NMIJ RM 8158-a.

\section{Acknowledgement}

This work was partly performed by the VAMAS TWA42 corresponding committee in Japan. We are grateful to Dr. Hyuksang Kwon (KRISS) for data collection with an $\mathrm{Ar}^{+}$laser, and to Dr. Masahiko 
Numata (NMIJ) for valuable discussions on developing the RM. 


\section{References}

1. I. R. Lewis and H. Edwards, "Handbook of Raman Spectroscopy Book Description Table of Contents", 2001, CRC Press, New York, 1072.

2. R. L. McCreery, "Raman Spectroscopy for Chemical Analysis", 2000, Wiley Interscence, NewYork, 420.

3. J. Ferraro, K. Nakamoto, and C. W. Brown, "Introductory Raman Spectroscopy", 2003, 2nd ed. , Academic Press, Amsterdam, 434.

4. E. Smith and G. Dent, “Modern Raman Spectroscopy - A Practical Approach”, 2005, Wiley, Chichester, 210.

5. ASTM E1840, "Standard Guide for Raman Shift Standards for Spectrometer Calibration".

6. N. Itoh, K. Shirono, and T. Fujimoto, Anal. Sci., 2019, 35, 571.

7. ISO Guide 35:2017, "Reference materials -- Guidance for characterization and assessment of homogeneity and stability".

8. J. A. Stone, J. E. Decker, P. Gill, P. Juncar, A. Lewis, G. D. Rovera, and M. Viliesid, Metrologia, 2009, 46, 11.

9. International Committee for weights and Measures (CIPM), "Recommended values of standard frequencies for applications including the practical realization of the metre and secondary representations of the second -Helium neon laser (unstabilized) ( $\mathrm{f} \approx 474 \mathrm{THz}$ )", 2007. Restrieved March 22, 2021 from https://www.bipm.org/utils/common/pdf/mep/M-eP_unstab-HeNe_633.pdf

10. E. B. Saloman and C. J. Sansonetti, J. Phys. Chem. Ref. Data, 2004, 33, 1113.

11. W. Whaling, W. H. C. Anderson, M. T. Carle, J. W. Brault, and H. A. Zarem, J. Res. Natl. Inst. Stand. Technol., 2002, 107, 149.

12. C. J. Sansonetti, J. Res. Natl. Inst. Stand. Technol., 2007, 112, 297.

13. National Institute of Metrology of China, "Certificate of GBW3654 (Acetoaminophen)", 
2018, Beijing.

14. E. B. Saloman, J. Phys. Chem. Ref. Data, 2010, 39. 


\section{Figure Caption}

\section{Figure 1.}

Raman spectrum of the polystyrene disc obtained under the same spectral and pixel resolutions for characterization. Peaks F, I, J, and K are magnified and inserted. The values in parentheses are the values described in ASTM E1840. ${ }^{5}$

\section{Figure 2.}

Uncertainty budget in the relative standard uncertainty for Raman shift of each peak. Value in the parenthesis is Raman shift $\left(\mathrm{cm}^{-1}\right)$ of each peak. 
Table 1. Summary of the measurement results

\begin{tabular}{|c|c|c|c|}
\hline Peak ID & Window No. & Wavenumber $\left(\mathrm{cm}^{-1}\right)$ & $u\left(\mathrm{~cm}^{-1}\right)$ \\
\hline \multirow[t]{3}{*}{$\mathrm{HeNe}$} & 1 & 15802.3235 & 0.0790 \\
\hline & 2 & 15801.8749 & 0.0532 \\
\hline & 3 & 15801.9024 & 0.1142 \\
\hline \multirow[t]{3}{*}{ Peak A } & 4 & 15181.6729 & 0.0530 \\
\hline & 5 & 15181.3054 & 0.0989 \\
\hline & 6 & 15181.6040 & 0.1313 \\
\hline \multirow[t]{3}{*}{ Peak B } & 4 & 15007.4012 & 0.0859 \\
\hline & 5 & 15006.7405 & 0.0848 \\
\hline & 6 & 15007.1313 & 0.1050 \\
\hline \multirow[t]{3}{*}{ Peak C } & 5 & 14800.7528 & 0.0931 \\
\hline & 6 & 14800.9476 & 0.1226 \\
\hline & 7 & 14800.3607 & 0.1058 \\
\hline \multirow[t]{3}{*}{ Peak D } & 5 & 14770.3575 & 0.0866 \\
\hline & 6 & 14770.5937 & 0.1273 \\
\hline & 7 & 14770.0386 & 0.1109 \\
\hline \multirow[t]{3}{*}{ Peak E } & 5 & 14647.1145 & 0.0899 \\
\hline & 6 & 14647.2173 & 0.1165 \\
\hline & 7 & 14646.5771 & 0.1083 \\
\hline \multirow[t]{4}{*}{ Peak F } & 6 & 14353.8383 & 0.0758 \\
\hline & 7 & 14352.9049 & 0.0946 \\
\hline & 8 & 14352.9448 & 0.1717 \\
\hline & 9 & 14352.7289 & 0.2215 \\
\hline \multirow[t]{3}{*}{ Peak G } & 7 & 14218.6548 & 0.0989 \\
\hline & 8 & 14218.7101 & 0.1555 \\
\hline & 9 & 14218.4389 & 0.2085 \\
\hline \multirow[t]{3}{*}{ Peak H } & 7 & 14199.2617 & 0.0989 \\
\hline & 8 & 14199.2808 & 0.1657 \\
\hline & 9 & 14199.0513 & 0.2055 \\
\hline \multirow[t]{3}{*}{ Peak I } & 10 & 12950.1328 & 0.0408 \\
\hline & 11 & 12950.1619 & 0.0490 \\
\hline & 12 & 12950.1050 & 0.0362 \\
\hline \multirow[t]{3}{*}{ Peak J } & 10 & 12895.0275 & 0.0491 \\
\hline & 11 & 12894.9566 & 0.0721 \\
\hline & 12 & 12894.7579 & 0.0803 \\
\hline \multirow[t]{3}{*}{ Peak K } & 10 & 12746.0021 & 0.0512 \\
\hline & 11 & 12745.9430 & 0.0364 \\
\hline & 12 & 12745.9512 & 0.0452 \\
\hline
\end{tabular}

Uncertainty $u$ is originated from ten measurements' reproducibility. 
Table 2a. Values with uncertainty from the literature and the results obtained for the Ne emission lines in Window 2

\begin{tabular}{|c|c|c|c|c|c|c|c|c|}
\hline \multirow[t]{2}{*}{ Line ID } & \multicolumn{2}{|c|}{$\begin{array}{l}\text { Reference wavenumber } \\
\text { in vacuum }\left(\mathrm{cm}^{-1}\right)^{* 1}\end{array}$} & \multicolumn{6}{|c|}{ Observed wavenumber in air $\left(\mathrm{cm}^{-1}\right)$} \\
\hline & $Y_{R e f ., 2}^{N e}$ & $u\left(Y_{R e f ., 2}^{N e}\right)$ & $X_{\text {Measure }, 2}^{N e}$ & $u\left(X_{\text {Measure }, 2}^{N e}\right)$ & $F_{\text {Fitting, } 2}^{N e}$ & $F_{\text {Repeat }, 2}^{N e}$ & $F_{\text {Repro.,i2 }}^{N e}$ & $u\left(Y_{\text {Measure. }, 2}^{N e}\right)$ \\
\hline $633 \mathrm{~nm}-4$ & 16458.1438 & 0.0014 & 16462.1406 & 0.1019 & - & 0.0065 & 0.0908 & 0.1367 \\
\hline $633 \mathrm{~nm}-5$ & 16399.2211 & 0.0013 & 16403.1980 & 0.0998 & - & 0.0053 & 0.0859 & 0.1318 \\
\hline $633 \mathrm{~nm}-6$ & 16274.021 & 0.0013 & 16277.9684 & 0.0954 & $9.095 \times 10^{-13}$ & 0.0041 & 0.0861 & 0.1285 \\
\hline $633 \mathrm{~nm}-7$ & 16219.8125 & 0.0013 & 16223.7473 & 0.1025 & $9.095 \times 10^{-13}$ & 0.0043 & 0.0884 & 0.1354 \\
\hline $633 \mathrm{~nm}-8$ & 16079.7523 & 0.0013 & 16083.6566 & 0.0988 & - & 0.0089 & 0.0836 & 0.1297 \\
\hline $633 \mathrm{~nm}-9$ & 15953.4708 & 0.0013 & 15957.3389 & 0.0964 & - & 0.0069 & 0.0869 & 0.1300 \\
\hline $633 \mathrm{~nm}-10$ & 15856.5732 & 0.0013 & 15860.4383 & 0.0909 & - & 0.0051 & 0.0826 & 0.1229 \\
\hline $633 \mathrm{~nm}-11$ & 15782.3821 & 0.0012 & 15786.2246 & 0.0929 & $9.095 \times 10^{-13}$ & 0.0038 & 0.0849 & 0.1259 \\
\hline $633 \mathrm{~nm}-12$ & 15662.3058 & 0.0012 & 15666.1379 & 0.0867 & - & 0.0057 & 0.0748 & 0.1147 \\
\hline $633 \mathrm{~nm}-13$ & 15615.197 & 0.0024 & 15619.0191 & 0.0831 & $9.095 \times 10^{-13}$ & 0.0051 & 0.0773 & 0.1136 \\
\hline $633 \mathrm{~nm}-14$ & 15364.9354 & 0.0012 & 15368.8556 & 0.0879 & - & 0.0049 & 0.0834 & 0.1212 \\
\hline $633 \mathrm{~nm}-15$ & 15302.9512 & 0.0012 & 15306.9325 & 0.0888 & - & 0.0025 & 0.0833 & 0.1218 \\
\hline
\end{tabular}

*1, Values and uncertainty were obtained from the literature. ${ }^{10}$ 
Table $2 \mathrm{~b}$. Values and uncertainty of reference data and the results obtained for the Ne emission lines in Window 5

\begin{tabular}{|c|c|c|c|c|c|c|c|c|}
\hline \multirow[t]{2}{*}{ Line ID } & \multicolumn{2}{|c|}{$\begin{array}{l}\text { Reference wavenumber } \\
\text { in vacuum }\left(\mathrm{cm}^{-1}\right)^{*_{1}}\end{array}$} & \multicolumn{6}{|c|}{ Observed wavenumber in air $\left(\mathrm{cm}^{-1}\right)$} \\
\hline & $Y_{R e f ., 5}^{N e}$ & $u\left(Y_{\text {Ref., }}^{N e}\right)$ & $X_{\text {Measure }, 5}^{N e}$ & $u\left(X_{\text {Measure }, 5}^{N e}\right)$ & $F_{\text {Fitting, } 5}^{N e}$ & $F_{\text {Repeat }, 5}^{N e}$ & $F_{\text {Repro.,5 }}^{N e}$ & $u\left(Y_{\text {Measure }, 5}^{N e}\right)$ \\
\hline $633 \mathrm{~nm}-14$ & 15364.9354 & 0.0012 & 15368.9730 & 0.0687 & - & 0.0214 & 0.0774 & 0.1057 \\
\hline $633 \mathrm{~nm}-15$ & 15302.9512 & 0.0012 & 15306.9826 & 0.0680 & - & 0.0208 & 0.0699 & 0.0997 \\
\hline $633 \mathrm{~nm}-16$ & 15149.7353 & 0.0011 & 15153.7002 & 0.0695 & - & 0.0218 & 0.0715 & 0.1020 \\
\hline $633 \mathrm{~nm}-17$ & 14969.7898 & 0.0011 & 14973.7114 & 0.0654 & - & 0.0207 & 0.0660 & 0.0951 \\
\hline $633 \mathrm{~nm}-18$ & 14883.3945 & 0.0011 & 14887.2924 & 0.0641 & $9.095 \times 10^{-13}$ & 0.0216 & 0.0635 & 0.0928 \\
\hline $633 \mathrm{~nm}-19$ & 14427.1441 & 0.0008 & 14431.2224 & 0.0616 & - & 0.0240 & 0.0703 & 0.0965 \\
\hline
\end{tabular}

*1, Values and uncertainty were obtained from the literature. ${ }^{10}$ 
Table 3a. Budget sheet for the HeNe laser in Windows 1, 2, and 3

\begin{tabular}{|c|c|c|c|c|c|c|c|c|}
\hline \multirow{2}{*}{ Parameter } & \multicolumn{2}{|c|}{ Window 1} & \multicolumn{2}{|c|}{ Window 2} & \multicolumn{2}{|c|}{ Window 3} & \multirow{2}{*}{ Unit } & \multirow{2}{*}{ Type } \\
\hline & Typical value & $u$ & Typical value & $u$ & Typical value & $u$ & & \\
\hline$a_{\mathrm{i}}$ & -4.2777 & 0.0423 & -3.8585 & 0.0549 & -3.6654 & 0.0507 & & A, B \\
\hline$b_{\mathrm{i}}$ & 1.0000 & $1.8892 \times 10^{-4}$ & 0.9998 & $2.6804 \times 10^{-4}$ & 0.9998 & $2.3075 \times 10^{-4}$ & & A, B \\
\hline$c_{\mathrm{i}}$ & $-4.9797 \times 10^{-7}$ & $2.1453 \times 10^{-7}$ & $-3.3121 \times 10^{-7}$ & $3.2347 \times 10^{-7}$ & $-4.5066 \times 10^{-7}$ & $3.2943 \times 10^{-7}$ & & A, B \\
\hline$d_{\mathrm{i}}$ & $2.2632 \times 10^{-11}$ & $6.6631 \times 10^{-10}$ & $5.6923 \times 10^{-10}$ & $1.0246 \times 10^{-9}$ & $4.6465 \times 10^{-10}$ & $1.0423 \times 10^{-9}$ & & A, B \\
\hline$X_{\text {Measure }, i}^{\text {HeNe }}$ & 15802.3235 & 0.0790 & 15801.8749 & 0.0532 & 15801.9024 & 0.1142 & $\mathrm{~cm}^{-1}$ & A \\
\hline$F_{\text {Fitting }, i}^{H e N e}$ & 1 & $2.9776 \times 10^{-8}$ & 1 & $4.3981 \times 10^{-8}$ & 1 & $1.6010 \times 10^{-8}$ & & A \\
\hline$F_{\text {Repeat }, i}^{\text {HeNe }}$ & 1 & $2.5663 \times 10^{-6}$ & 1 & $2.9621 \times 10^{-6}$ & 1 & $6.1812 \times 10^{-7}$ & & A \\
\hline$F_{\text {Repro.,i }}^{\text {HeNe }}$ & 1 & $6.6461 \times 10^{-6}$ & 1 & $6.5795 \times 10^{-6}$ & 1 & $8.9626 \times 10^{-6}$ & & A \\
\hline$Y_{\text {Calculation }, i}^{\text {HeNe }}$ & 15797.9737 & 0.1686 & 15798.0365 & 0.1406 & 15798.1249 & 0.2138 & $\mathrm{~cm}^{-1}$ & \\
\hline
\end{tabular}


Table 3b. Budget sheet for Peak $\mathrm{C}$ in Windows 5, 6, and 7

\begin{tabular}{|c|c|c|c|c|c|c|c|c|}
\hline \multirow{2}{*}{ Parameter } & \multicolumn{2}{|c|}{ Window 5} & \multicolumn{2}{|c|}{ Window 6} & \multicolumn{2}{|c|}{ Window 7} & \multirow{2}{*}{ Unit } & \multirow{2}{*}{ Type } \\
\hline & Typical value & $u$ & Typical value & $u$ & Typical value & $u$ & & \\
\hline$a_{\mathrm{i}}$ & -3.9275 & 0.0628 & -4.0643 & 0.0846 & -3.3119 & 0.0873 & & A, B \\
\hline$b_{\mathrm{i}}$ & 0.9997 & $5.3345 \times 10^{-4}$ & 0.9998 & $3.3863 \times 10^{-4}$ & 1.0000 & $8.5861 \times 10^{-4}$ & & A, B \\
\hline$c_{\mathrm{i}}$ & $-4.2415 \times 10^{-7}$ & $1.0585 \times 10^{-6}$ & $-6.3806 \times 10^{-7}$ & $4.2466 \times 10^{-7}$ & $-6.9916 \times 10^{-7}$ & $8.0452 \times 10^{-7}$ & & $\mathrm{~A}, \mathrm{~B}$ \\
\hline$d_{\mathrm{i}}$ & $8.4645 \times 10^{-10}$ & $3.0535 \times 10^{-9}$ & $5.6666 \times 10^{-10}$ & $1.3489 \times 10^{-9}$ & $5.6866 \times 10^{-10}$ & $3.9800 \times 10^{-9}$ & & $\mathrm{~A}, \mathrm{~B}$ \\
\hline$X_{\text {Measure }, i}^{\text {Raman }}$ & 14800.7528 & 0.0931 & 14800.9476 & 0.1226 & 14800.3607 & 0.1058 & $\mathrm{~cm}^{-1}$ & A \\
\hline$F_{\text {Fitting }, i}^{\text {Raman }}$ & 1 & $2.2399 \times 10^{-7}$ & 1 & $1.8400 \times 10^{-7}$ & 1 & $5.0150 \times 10^{-8}$ & & A \\
\hline$F_{\text {Repeat }, i}^{\text {Raman }}$ & 1 & $8.9754 \times 10^{-7}$ & 1 & $8.9756 \times 10^{-7}$ & 1 & $1.8921 \times 10^{-6}$ & & A \\
\hline$F_{\text {Repro.,i }}^{\text {Raman }}$ & 1 & $5.1809 \times 10^{-6}$ & 1 & $5.0415 \times 10^{-6}$ & 1 & $4.6774 \times 10^{-6}$ & & A \\
\hline$Y_{\text {Calculation }, i}^{\text {Raman }}$ & 14796.8575 & 0.1900 & 14796.8702 & 0.1708 & 14796.9392 & 0.6035 & $\mathrm{~cm}^{-1}$ & \\
\hline
\end{tabular}


Table 4. Combined wavenumbers and their uncertainty for each peak, as well as the uncertainties considered

\begin{tabular}{|c|c|c|c|c|c|c|c|c|}
\hline \multirow{2}{*}{ Laser/Peak ID } & \multirow{2}{*}{ Typical Value } & \multicolumn{3}{|c|}{ Combining windows } & \multirow{2}{*}{$u_{H e N e}$} & \multirow{2}{*}{$u_{\text {Pixel }}$} & \multirow{2}{*}{$u_{\text {Resolution }}$} & \multirow{2}{*}{$u_{c}^{\text {Wavenumber }}$} \\
\hline & & $u_{\text {Window }}$ & $u_{b w}$ & $u_{c}^{\text {Window }}$ & & & & \\
\hline $\mathrm{HeNe}$ & 15798.0388 & 0.0977 & - & 0.0977 & 0.0237 & 0.3738 & - & 0.3871 \\
\hline Peak A & 15177.3122 & 0.1106 & 0.0508 & 0.1217 & - & 0.3450 & - & 0.3658 \\
\hline Peak B & 15002.8973 & 0.1169 & 0.0687 & 0.1356 & - & 0.3363 & - & 0.3626 \\
\hline Peak C & 14796.8739 & 0.1356 & 0.0301 & 0.1389 & - & 0.3272 & - & 0.3554 \\
\hline Peak D & 14766.5144 & 0.1379 & 0.0777 & 0.1583 & - & 0.3257 & - & 0.3621 \\
\hline Peak E & 14643.1815 & 0.1546 & - & 0.1546 & - & 0.3199 & - & 0.3553 \\
\hline Peak F & 14349.6057 & 0.1735 & 0.0430 & 0.1788 & - & 0.3064 & 0.1021 & 0.3691 \\
\hline Peak G & 14215.2956 & 0.1461 & - & 0.1461 & - & 0.3012 & - & 0.3347 \\
\hline Peak H & 14195.9717 & 0.1456 & 0.0279 & 0.1483 & - & 0.3002 & - & 0.3348 \\
\hline Peak I & 12947.0352 & 0.0961 & - & 0.0961 & - & 0.2483 & - & 0.2662 \\
\hline Peak J & 12891.8203 & 0.1127 & 0.0714 & 0.1334 & - & 0.2483 & 0.0982 & 0.2985 \\
\hline Peak K & 12742.9163 & 0.0837 & 0.0877 & 0.1212 & - & 0.2401 & 0.0671 & 0.2772 \\
\hline
\end{tabular}


Table 5. Reference values and their expanded uncertainties

\begin{tabular}{lccc}
\hline Peak ID & $\begin{array}{c}\text { Raman shift } \\
\left(\mathrm{cm}^{-1}\right)\end{array}$ & $\begin{array}{c}\text { Coverage } \\
\text { factor }\end{array}$ & $\begin{array}{c}U \\
\left(\mathrm{~cm}^{-1}\right)\end{array}$ \\
\hline \hline Peak A & 620.7 & 2 & 1.2 \\
Peak B & 795.1 & 2 & 1.2 \\
Peak C & 1001.2 & 2 & 1.2 \\
Peak D & 1031.5 & 2 & 1.2 \\
Peak E & 1154.9 & 2 & 1.2 \\
Peak F & 1448.4 & 2 & 1.2 \\
Peak G & 1582.7 & 2 & 1.2 \\
Peak H & 1602.1 & 2 & 1.2 \\
Peak I & 2851.0 & 2 & 1.1 \\
Peak J & 2906.2 & 2 & 1.2 \\
Peak K & 3055.1 & 2 & 1.1 \\
\hline
\end{tabular}




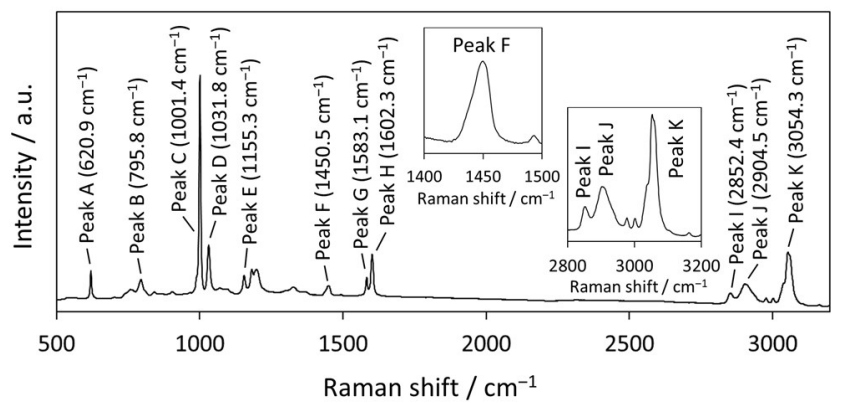

Figure 1.

Raman spectrum of the polystyrene disc obtained under the same spectral and pixel resolutions for characterization. Peaks F, I, J, and $\mathrm{K}$ are magnified and inserted. The values in parentheses are the values described in ASTM E1840. ${ }^{5}$ 


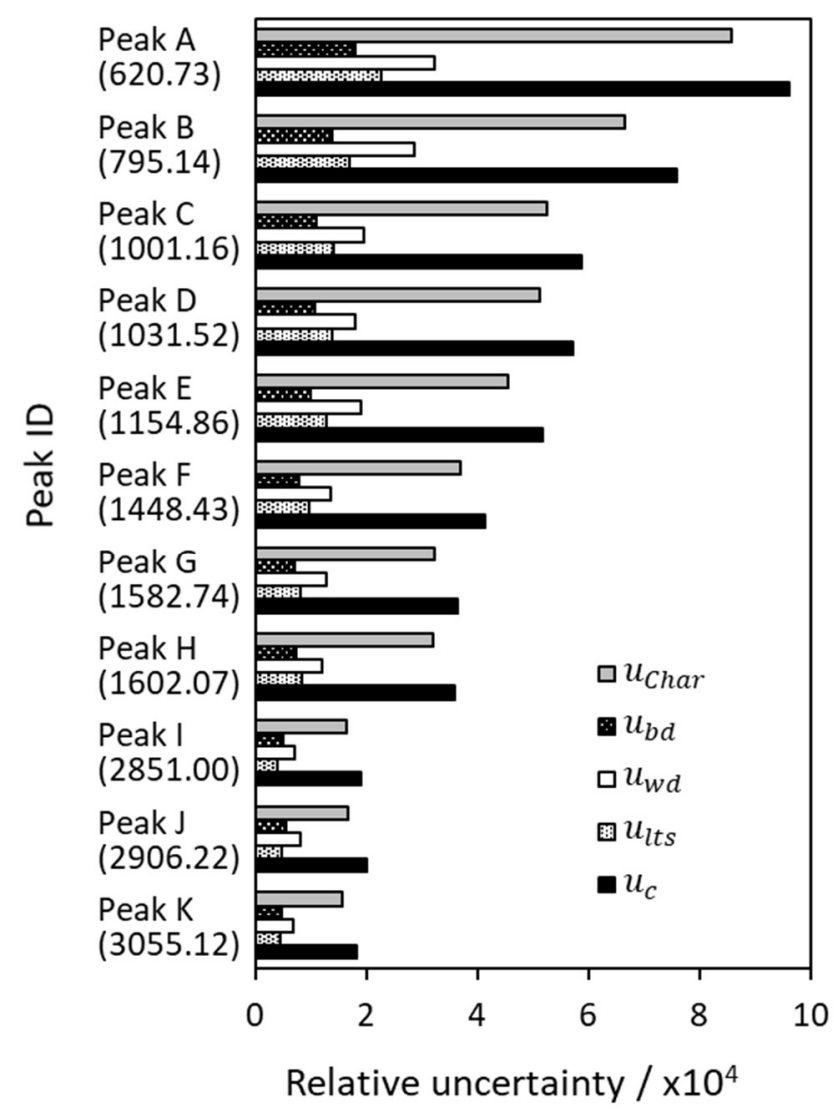

Figure 2.

Uncertainty budget in the relative standard uncertainty for Raman shift of each peak. Value in the parenthesis is Raman shift $\left(\mathrm{cm}^{-1}\right)$ of each peak. 


\section{Graphical Index}

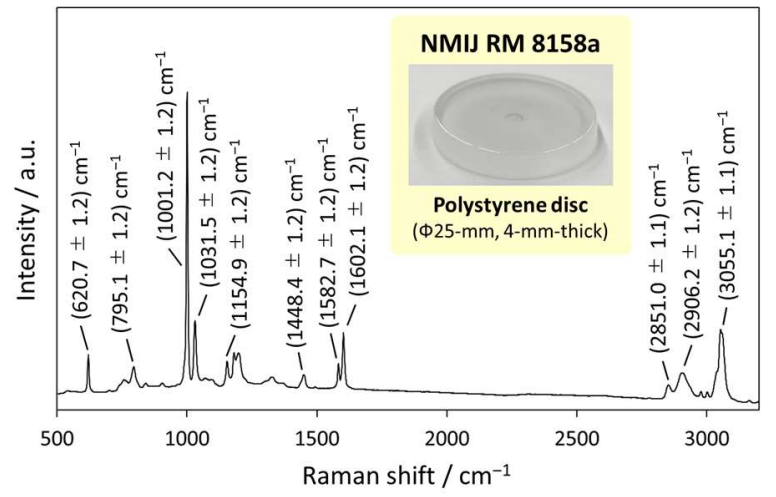

